\title{
Elevation of Autoantibody in Patients with Ischemic Stroke
}

\author{
Yoichi YoshIDA, ${ }^{1,2,3}$ Takaki HiWASA, ${ }^{2}$ Toshio MACHIDA, ${ }^{4,5}$ Eiichi KoBAYASHI, ${ }^{1,3}$ \\ Seiichiro Mine, ${ }^{4,6}$ Jun MATSUSHIMA, ${ }^{7}$ Masaki TAKIGUCHI, ${ }^{2}$ and Yasuo IWADATE ${ }^{1}$
}

\author{
${ }^{1}$ Department of Neurological Surgery, Graduate School of Medicine, \\ Chiba University, Chiba, Chiba, Japan; \\ ${ }^{2}$ Department of Biochemistry and Genetics, Graduate School of Medicine, \\ Chiba University, Chiba, Chiba, Japan; \\ ${ }^{3}$ Comprehensive Stroke Center, Chiba University Hospital, \\ Chiba, Chiba, Japan; \\ ${ }^{4}$ Department of Neurosurgery, Chiba Cerebral and Cardiovascular Center, \\ Ichihara, Chiba, Japan; \\ ${ }^{5}$ Department of Neurosurgery, Eastern Chiba Medical Center, Tougane, Chiba, Japan; \\ ${ }^{6}$ Department of Neurosurgery, Prefectural Sawara Hospital, Katori, Chiba, Japan; \\ ${ }^{7}$ Department of Diagnostic Pathology, Graduate School of Medicine, Chiba \\ University, Chiba, Chiba, Japan
}

\begin{abstract}
Recent clinical research has revealed a significant correlation between atherosclerosis, one of the primary etiologies of ischemic stroke, and the immune system. Assuming that "disease-specific autoantibodies are produced in the sera of patients with ischemic stroke," we investigated multiple arteriosclerosis-related antibodies using the serological identification of antigens by recombinant cDNA expression cloning (SEREX), an established method for identifying antigenic proteins. We either screened a human aortic endothelial cell cDNA library or conducted protein array screening using the sera from patients with ischemic stroke, such as carotid artery stenosis or transient ischemic attack (TIA). Next, we measured serum antibody levels using amplified luminescent proximity homogeneous assay-linked immunosorbent assay (AlphaLISA) in patient/healthy donor (HD) cohorts and identified several antigens, the antibody levels of which were significantly higher in patients with ischemic stroke than in HDs. This review introduced the method of identifying antigens by the SEREX and protein microarray and summarized antigenic proteins. In particular, it focused on anti-replication protein A2 antibody and anti-programmed cell death 11 antibody, which are significantly related to atherosclerotic plaque and ischemic brain tissue, respectively, and proposed the mechanism of elevated autoantibody levels against them. Furthermore, this review suggests a possibility of clinical application as an atherosclerotic disease diagnostic marker for TIA or cerebral infarction.
\end{abstract}

Key words: autoantibody, TIA, cerebral infarction, biomarker, atherosclerosis

\section{Introduction}

Ischemic stroke is one of the leading causes of morbidity and mortality worldwide, with arteriosclerosis as one of its primary etiologies, the suppression of which is imperative for the prevention of onset. ${ }^{1-3)}$ To date, several factors have been recognized as underlying the progress of arteriosclerosis, such as age, high blood pressure, hyperlipidemia, diabetes, smoking, and obesity. ${ }^{4)}$ However, in recent years,

Received January 25, 2018; Accepted March 20, 2018

Copyright $\subset 2018$ by The Japan Neurosurgical Society This work is licensed under a Creative Commons AttributionNonCommercial-NoDerivatives International License. some studies have highlighted that the immune system is profoundly involved in the progression of arteriosclerosis. ${ }^{5-9)}$ Assuming that "disease-specific autoantibodies are produced in the sera of patients with ischemic stroke," we investigated multiple arteriosclerosis-related antibodies using the serological identification of antigens by recombinant cDNA expression cloning (SEREX), an established method for identifying antigenic proteins. ${ }^{10,11)}$

Of these antibodies, we focused on anti-replication protein A2 (RPA 2) antibody and anti-programmed cell death 11 (PDCD 11) antibody, which were significantly related to atherosclerotic plaque and ischemic brain tissue, respectively. RPAs are composed of three subunit proteins of 70, 32, and $14 \mathrm{kDa}$ (RPA1, 
RPA2, and RPA3, respectively). In the rat brain, DNA double-strand breaks (DSBs) were induced by ischemia, ${ }^{12)}$ and BRCA1 which is involved in the repair of DNA DSBs has been shown to be involved in heart function and survival following myocardial infarction. ${ }^{13)}$ It can be speculated that the RPA complex works in the upstream pathway of BRCA1 in the DSB repair pathway. ${ }^{14,15)}$ In atherosclerotic plaque, cells are exposed to reactive oxygen species, which can provoke extensive oxidative DNA damage, ${ }^{16,17)}$ then; the RPA complex which is required for DSB repair might be overexpressed in atherosclerotic lesion. On the other hand, PDCD11 is a NF- $\mathrm{KB}-$ binding protein that colocalizes with U3 RNA in the nucleolus and is required for rRNA maturation and generation of 18S rRNA. PDCD11 is necessary for Fas ligand (FasL) expression, and PDCD11 overexpression is known to induce transcription of FasL, leading to the induction of apoptosis through Fas/ FasL/caspase death pathway. ${ }^{18-21)}$ It is also reported that post-stroke inflammatory response of FasL is an important contributing mechanism in ischemic brain lesion. ${ }^{22)}$ Therefore, it can be speculated that Fas/FasL/caspase death pathway may be activated in the ischemic brain tissue and that PDCD11 may be overexpressed.

This review introduces the method of identifying antigens by the SEREX and protein microarray and summarizes antigenic proteins. In addition, it discusses a proposed mechanism of the elevation of autoantibody and a possibility of clinical application as a disease diagnostic marker.

\section{Autoantibody against Atherosclerotic Lesion}

\section{Immune system associated with atherosclerosis}

In the arteriosclerotic lesions, several histopathological studies have observed macrophage and lymphocyte infiltration simultaneously as the deposition of lipid in the subintimal membrane, ${ }^{23)}$ suggesting that the immune system is intensely involved in the establishment of arteriosclerosis.

Apparently, macrophages release several substances, such as free radicals and various proteases, to induce tissue damage and secrete fibrogenic cytokines and play a central role in the formation of advanced arteriosclerosis, such as fibrous thickening of the intima and remodeling of vascular architecture. ${ }^{24)}$ In atherosclerotic lesions, T-lymphocytes and vascular dendritic cells are reportedly involved in macrophages activation, ${ }^{23,25)}$ suggesting the presence of an immune mechanism strongly through antigen presentation for arteriosclerosis.
Several studies have been conducted on antigens related to the occurrence of arteriosclerosis, ${ }^{26)}$ and oxidized low-density lipoproteins (oxLDL) constitute a typical example for candidate antigens. Autoantibodies against oxLDL are detected in the circulating blood, as well as atherosclerotic lesions locally; research has reported a correlation between their autoantibody levels and the degree of arteriosclerosis. In addition, many studies have reported that autoantibodies against heat shock protein (Hsp) family, such as Hsp 27, 60, or 70, are elevated in patients with arteriosclerotic diseases. Furthermore, it is suggested that cross-immunization with bacteriaderived Hsp is its pathogen.

\section{SEREX}

The SEREX, a combination of molecular cloning using phage expression libraries with serological typing, is an established method for comprehensively recognizing cancer-specific antigenic proteins and was first reported by Sahin et al. ${ }^{10)}$ First, mRNA was extracted from a cancer tissue to prepare a cDNA library, which was incorporated into the DNA of the phage vector for protein expression. Next, Escherichia coli were infected with the phage, and the expression of resident cDNA clones was induced after blotting inflectional bacteria onto the nitrocellulose membrane. Membranes were then incubated with the sera of patients. Finally, cancer antigens were recognized in patients' immune system by collecting clones that exhibited a positive reaction and analyzing the base sequences.

\section{Protein microarray screening}

In addition to the SEREX, we used the ProtoArray v-4.0 human protein microarray system as another screening method, ${ }^{27)}$ wherein we extracted antigen proteins identified by IgG autoantibodies in the sera of patients. This method identified more candidate antigenic proteins.

\section{Second screening by AlphaLISA}

Although the SEREX screening comprehensively recognized positive clones accountable for specific diseases, it could not quantify autoantibody levels in the sera of patients. Next, we compared the serum antibody levels against the candidate antigenic protein using the sera of patients and healthy donors (HDs) to ascertain a relationship between candidate antigens and diseases. We used amplified luminescent proximity homogeneous assay-linked immunosorbent assay (AlphaLISA) for evaluating the serum antibody level. AlphaLISA is an immunoassay using anti-human IgG-conjugated acceptor 
beads and glutathione-conjugated donor beads, in which the antigen-antibody reaction in the serum and antigenic protein substitute with luminescence because of a chemical reaction between these beads and is detected with a dedicated measuring instrument; this luminescence reflects the antibody level. Finally, antigens with antibody levels by AlphaLISA significantly higher in patients than in HDs were determined as disease-related antigens.

Identified antigens associated with ischemic stroke

The SEREX is one of the most effective methods for identifying antigenic targets on a genomic scale and has been used to identify more than 1,000 novel cancer antigens. ${ }^{28-30)}$ Despite initially being developed to screen cancer-associated antigens, this method has been applied for vascular disorders, such as transplant-associated coronary disease, ${ }^{31)}$ Kawasaki disease, ${ }^{32)}$ and Moyamoya disease. ${ }^{33)}$ Thus, we conducted the SEREX screening using a commercially available human aortic endothelial cell cDNA library and the sera of patients, followed by the identification of several antigens accountable for atherosclerotic diseases, such as cerebral infarction, cardiovascular disease (CVD), and diabetes mellitus (DM). ${ }^{34-40)}$ Of these antigens, we introduced antigens screened using the sera of patients with carotid artery stenosis and transient ischemic attack (TIA).

\section{Identified associated antigens responsible for ischemic stroke}

First, we screened a human aortic endothelial cell cDNA library using the sera of patients with ischemic stroke due to severe ( $>90 \%$ ) carotid stenosis. Among isolated candidates in the SEREX screening, RPA2, ${ }^{34)}$ TUBB2C, ${ }^{35)}$ ATP2B4, and BMP-1 ${ }^{36)}$ were considered to be associated with ischemic stroke, as proven in patient/HD cohorts by AlphaLISA. Subsequently, we performed immunological screening using the sera of patients with TIA and considered PDCD11, ${ }^{37}$ MMP1, CBX1, and $\mathrm{CBX}^{38)}$ to be associated with cerebral infarction or TIA. In addition, we performed protein microarray screening and recognized DHPS $^{39)}$ and $\mathrm{SH}_{3 \mathrm{BP}}{ }^{40)}$ to be associated with ischemic stroke, as demonstrated in patient/HD cohorts.

Compared to HDs, serum antibody levels against RPA2 (RPA2-Ab levels) were significantly higher in patients with ischemic stroke. Thus, we further investigated RPA2-Ab levels using synthetic peptides by enzyme-linked immunosorbent assay to identify the antigenic epitopes of RPA2. ${ }^{34)}$ In this study, the sensitivity and specificity with autoantibody levels against peptide bRPA2-132 (biotin-LRSFQNKKSLVAFKI; amino acids between positions 132 and 146) were higher than those with RPA2Ab. Next, we assessed antibody levels against peptide antigen of RPA2-132 (bRPA2-Ab levels) in the following study. Similarly, serum autoantibody levels against TUBB2C were significantly higher in patients with cerebral infarction than in HDs. Furthermore, autoantibody levels were elevated in patients with CVD and DM. ${ }^{35)}$ Similar results were obtained with anti-ATP2B4 antibody, anti-BMP-1 antibody, anti-DHPS antibody, and anti-SH3BP5 antibody. ${ }^{36,39,40)}$ Incidentally, these autoantibody levels were elevated not only in patients with cerebral infarction but also in patients with TIA. Conversely, serum autoantibody levels against PDCD11 (PDCD11-Ab levels) and CBX1, which were screened using the sera of patients with TIA, were significantly higher in patients with cerebral infarction or TIA than in HDs. However, autoantibody levels were not elevated in patients with acute myocardial infarction (AMI) and DM. ${ }^{37,38)}$ Table 1 summarizes the above results.

Therefore, various antigenic proteins considered responsible for ischemic stroke differed in results, resulting in speculation that the mechanism of autoantibody production correlated with this background of difference. An immunohistological examination on RPA 2 and PDCD 11 revealed that RPA 2 was

Table 1 Association with autoantibody biomarkers and atherosclerotic diseases

\begin{tabular}{|c|c|c|c|}
\hline & CI & $\mathrm{DM}$ & CVD \\
\hline Replication protein A2 (RPA2) ${ }^{23)}$ & $\uparrow$ & $\uparrow$ & $\uparrow$ \\
\hline Tublin beta 2C (TUBB2C) ${ }^{24)}$ & $\uparrow$ & $\uparrow$ & $\uparrow$ \\
\hline $\begin{array}{l}\text { ATPase, } \mathrm{Ca}^{++} \text {transporting, } \\
\text { plasma membrane } 4(\text { ATP2B } 4)^{25)}\end{array}$ & $\uparrow$ & $\uparrow$ & $\uparrow$ \\
\hline $\begin{array}{l}\text { Bone morphogenetic protein } 1 \\
(\text { BMP-1 })^{25)}\end{array}$ & $\uparrow$ & $\uparrow$ & $\uparrow$ \\
\hline $\begin{array}{l}\text { Matrix metalloproteinase } 1 \\
(\mathrm{MMP} 1)^{27)}\end{array}$ & $\uparrow$ & $\uparrow$ & $\uparrow$ \\
\hline $\begin{array}{l}\text { Deoxyhypusine synthase } \\
\text { (DHPS) }^{28)}\end{array}$ & $\uparrow$ & $\uparrow$ & $\uparrow$ \\
\hline $\begin{array}{l}\text { SH3 domain-binding protein } 5 \\
(\text { SH3BP5) }\end{array}$ & $\uparrow$ & $\uparrow$ & $\uparrow$ \\
\hline $\begin{array}{l}\text { Programed cell death } 11 \\
\text { (PDCD11) }^{26)}\end{array}$ & $\uparrow$ & - & - \\
\hline Chromobox homolog $1(\mathrm{CBX} 1)^{27)}$ & $\uparrow$ & - & - \\
\hline
\end{tabular}

We examined autoantibody levels against above antigens in patients with atherosclerotic diseases, such as cerebral infarction (CI), diabetes mellitus (DM), and cardiovascular disease (CVD). “个” showed that serum antibody levels were significantly higher in patients compared to those in healthy donors (HDs). On the other hand, "-" showed that serum antibody levels were not elevated in patients. We added the number of reference. 

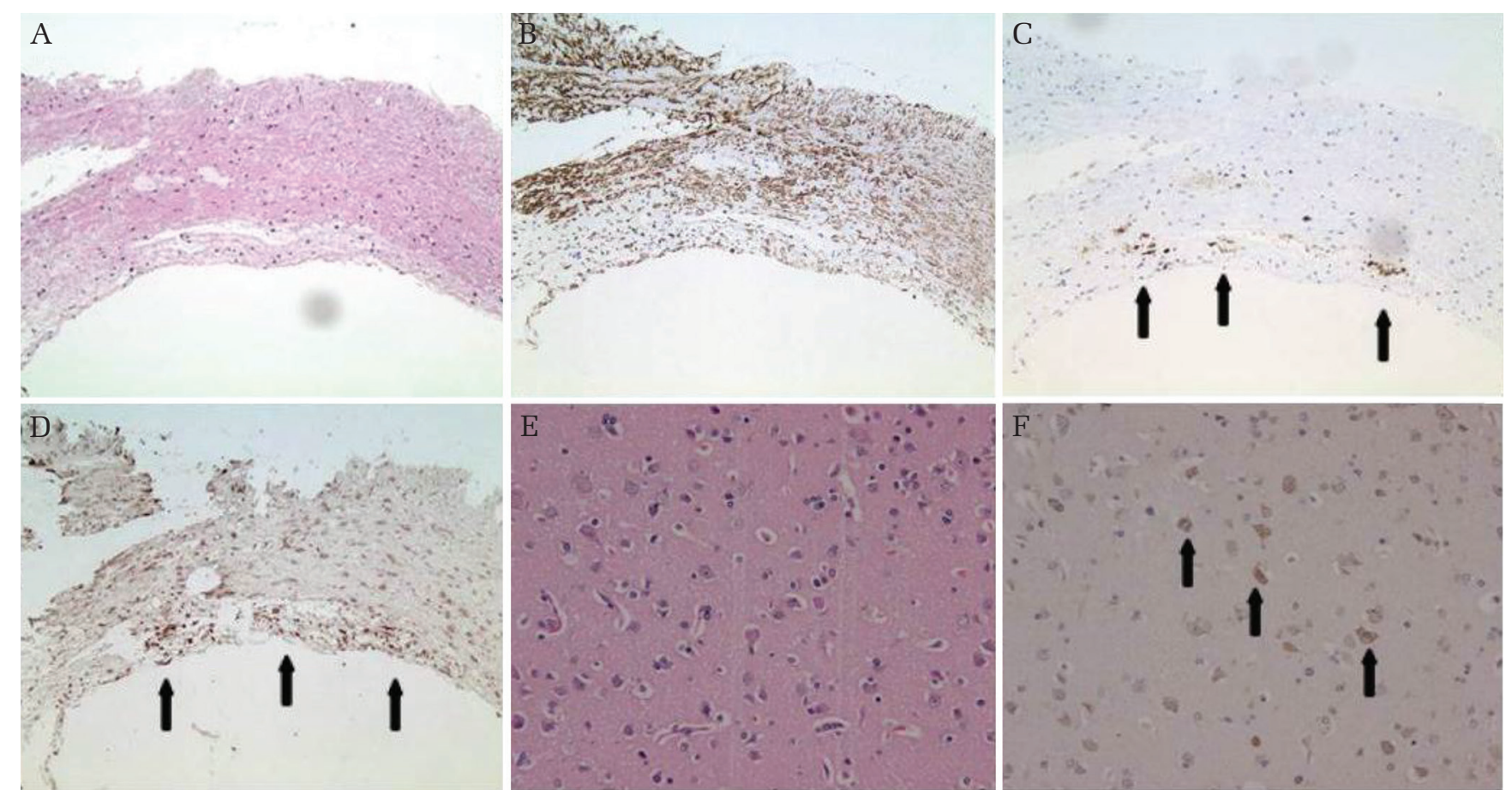

Fig. 1 Immunohistochemistry. Surgically excised carotid plaque (A-D) was stained with hematoxylin only (A), anti-SMC; which identified vascular smooth muscle cells (B), anti-CD68; which identified macrophages (C, arrow), or anti-RPA2 antibody (D, arrow). Surgically resected ischemic brain tissue (E and F) was stained with hematoxylin only (E) and anti-PDCD11 antibody (F, arrow). Photos reprinted with the permission of BioMed Central journals (A-D) and Oncotarget ( $E$ and $F)$.

significantly expressed in the intima of arteriosclerotic plaque (Figs. 1A-1D), ${ }^{34)}$ resulting in the autoantibody production. In contrast, it was confirmed that the PDCD11 expression was closely co-localized with ischemic brain cells (Figs. 1E and 1F), ${ }^{37)}$ and this expression was considered to have led to the production of PDCD11 autoantibody through an autoimmune response.

As mentioned above, it could be considered that while the elevation of serum RPA2-Ab levels reflects the presence of arteriosclerotic lesions, the elevation of serum PDCD11-Ab levels reflects the presence of a cerebral ischemic lesion, thereby both having different autoantibody production mechanisms. In addition, this finding verified the reason why serum PDCD11-Ab levels were not elevated in patients with AMI and DM. Instead, it was considered that the elevation of serum RPA2-Ab levels reflecting the arteriosclerotic lesion was also observed in other arteriosclerotic diseases. In fact, we investigated correlations between bRPA2-Ab levels and AMI and DM in another validation cohort using the sera of each 128 age-matched patients and HDs. AlphaLISA revealed statistically significantly higher bRPA2-Ab levels in patients with AMI $(P<0.001)$ or DM $(P<0.001)$ compared to those in HDs (Fig. 2, Table 1).

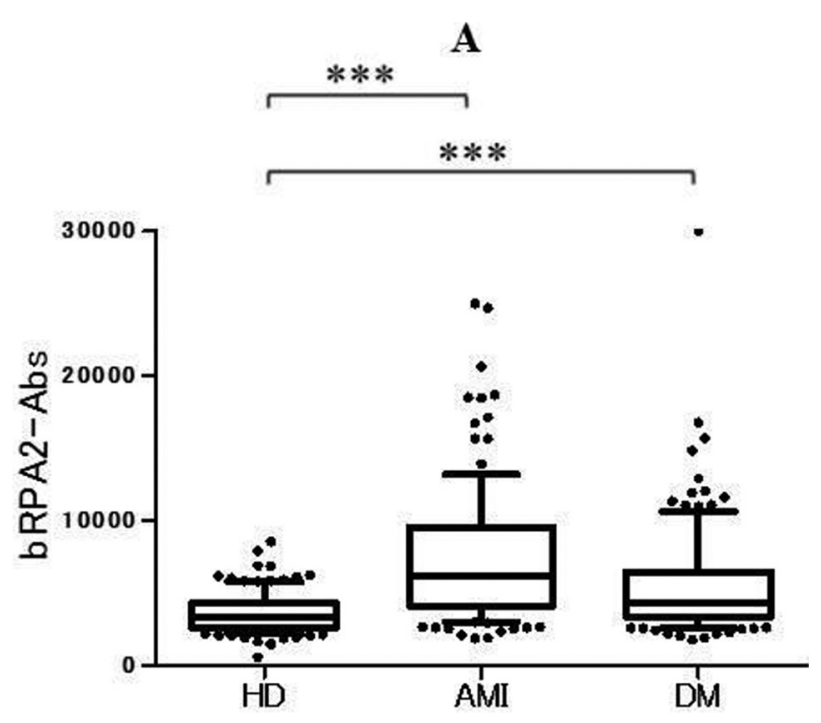

Fig. 2 The correlation between autoantibody levels and other atherosclerotic diseases, including AMI and DM. The levels of bRPA2-Ab (A) were examined by AlphaLISA. The mean age \pm SDs of HDs and patients with AMI or $\mathrm{DM}$ were $58.29 \pm \mathbf{5 . 6 3}, \mathbf{5 8 . 2 8} \pm \mathbf{8 . 5}$, and $58.37 \pm \mathbf{9 . 1 1}$ years, respectively. ${ }^{* *} \boldsymbol{P}<\mathbf{0 . 0 0 1}$ was calculated by the Mann-Whitney $U$-test with type I error adjustment using the Bonferroni procedure. bRPA2-Ab, antibody against peptide antigen of RPA2-132; AMI, acute myocardial infarction; DM, diabetes mellitus; HD, healthy donors. 


\section{Utility of identified autoantibody as a diagnostic marker}

The determination of a disease-specific elevation in the autoantibody level suggests the involvement of some form of immune system in the pathology of the disease and elucidating its mechanism might illustrate the pathology of the disease. Conversely, the elevation in serum disease-specific antibody levels could be a potential disease diagnostic marker.

Furthermore, it is likely that antigen proteins leak out from vascular lesion by recurrent microenvironmental artery destruction, which frequently occurs before the onset of ischemic stroke. Although the antigen proteins disappeared rapidly, antibodies can be amplified after repeated exposure to antigen proteins. Based on this perspective, antibody markers might be more sensitive than antigen markers because repeated exposure of a small amount of an antigen to immune cells leads to the production of a large number of antibodies. Thus, increasing the number of antibody markers, such as those against oxidized LDL for atherosclerosis, ${ }^{411}$ p53 antibodies for cancer, ${ }^{42)}$ and glutamic acid decarboxylase antibodies for type-1 $\mathrm{DM},{ }^{43)}$ has been recommended for practical use.

Transient ischemic attack was one of the significant risk factors for ischemic stroke, and approximately $15-20 \%$ of patients with ischemic stroke are estimated to experience TIA before the onset of a stroke. ${ }^{44,45)}$ Recent clinical studies have investigated early intervention benefits in patients with TIA to prevent the subsequent development of cerebral infarction. ${ }^{46)}$ However, the diagnosis of TIA is occasionally challenging because physicians have to diagnose TIA only by considering history and they have few objective pieces of evidence detected by various medical examinations, such as magnetic resonance imaging, echocardiogram, and Holter electrocardiogram. Hence, the diagnosis of TIA simply by blood examination will significantly contribute to the clinical practice in preventing stroke development, as well as facilitating medical economy. Despite highlighting that the elevated PDCD11-Ab level was an independent predictor of TIA, unfortunately, the diagnostic value of PDCD11 alone was weak (sensitivity and specificity were $73.6 \%$ and $55.8 \%$, respectively). ${ }^{37)}$

We believe that the diagnostic value will increase by a combination of the measurement of multiple autoantibodies with the variable mechanism of production. Next, we examined serum bRPA2-Ab and PDCD11-Ab levels using the sera of 92 patients with TIA and 285 HDs; either bRPA2-Ab or PDCD11-Ab levels were elevated in $79.3 \%$ of patients with TIA,
Table 2A Positive predictive values for TIA $(n=92)$

\begin{tabular}{llrcc}
\hline & & \multicolumn{2}{c}{ bRPA2 $(>1731)^{*}$} & \multirow{2}{*}{ Total } \\
\cline { 3 - 4 } & & Positive & Negative & \\
\hline PDCD11 & Positive & 35 & 33 & 68 \\
$(>13,921)^{* *}$ & Negative & 5 & 19 & 24 \\
\multicolumn{2}{c}{ Total } & 40 & 52 & 92 \\
\hline
\end{tabular}

Table 2B Negative predictive values for HDs $(n=285)$

\begin{tabular}{llccc}
\hline \multirow{2}{*}{ Positive } & & \multicolumn{2}{c}{ bRPA2 $(>1731)^{*}$} & \multirow{2}{*}{ Total } \\
\cline { 3 - 4 } & & Positive & Negative & \\
\hline PDCD11 & Positive & 63 & 63 & 126 \\
$(>13,921)^{* *}$ & Negative & 27 & 132 & 159 \\
\multicolumn{2}{c}{ Total } & 90 & 195 & 285 \\
\hline
\end{tabular}

Either bRPA2-Ab or PDCD11-Ab levels were elevated in 73 patients with TIA $(n=92)$. Both of antibody levels were not elevated in 19 patients with TIA and 132 HDs (total = 151).

*RPA2, elevated RPA2-Ab levels. RPA2-Ab cut-off was 1731 based on ROC curve analysis. **PDCD11, elevated PDCD11Ab levels. PDCD11-Ab cut-off was 13921 based on ROC curve analysis.

and $87.4 \%$ of those who were both of antibody levels were not elevated was HDs (Tables 2A and 2B). Assumedly, this diagnostic value was superior to diagnosis by a single marker. In addition, we are now attempting to produce a peptide antigen of PDCD11 with a localized epitope, which might enhance the sensitivity and specificity. Moreover, the finding that the diagnostic value can increase by a combination of the measurement of multiple autoantibodies with difference production mechanism led us to anticipate the possibility that the diagnostic value will be further enhanced by novel combining markers in the future. Furthermore, it was documented that positive predictive values for TIA with the combination of PDCD11-Ab and clinical risk factors were higher than those with clinical risk factors alone, and all individuals with these four factors developed TIA, including age, hypertension, diabetes, and elevated PDCD11Ab levels. ${ }^{37)}$ Thus, the evaluation of antibody markers along with clinical factors might be useful for diagnosis.

\section{Conclusion}

This review introduced the ischemic stroke-related antigens determined using the SEREX, particularly, the antibody against RPA2 and PDCD11 implying the arteriosclerotic lesion and the cerebral ischemic tissues, respectively. In addition, it revealed that the elevation of the serum PDCD11-Ab level was an independent predictor of TIA. Thus, combining autoantibody markers with various mechanisms of 
production might increase the diagnostic value and be valuable for the estimation of the risk of developing atherosclerotic cerebral infarction.

\section{Ethical Approval}

This study was approved by the Local Ethical Review Board of the Graduate School of Medicine, Chiba University, as well as those of co-operating hospitals, and it was performed in accordance with the principles of the Declaration of Helsinki. Written informed consents were obtained from all participants. Recombinant DNA studies were performed with official permission No. 251 from the Graduate School of Medicine, Chiba University, and conducted in conformity with the rules of the Japanese government.

\section{Author Contributions}

Yoichi Yoshida carried out the experiments, performed the statistical analysis and drafted the manuscript. Takaki Hiwasa conceived of the study, and participated in its design and coordination and helped to draft the manuscript. Toshio Machida performed the screening by expression cloning and helped to draft the manuscript. Eiichi Kobayashi, Masaki Takiguchi, and Yasuo Iwadate participated in the design of the study. Seiichiro Mine collected the blood sampling and performed the statistical analysis. Jun Matsushima helped to perform the immunohistochemistry. All authors read and approved the final manuscript.

\section{Acknowledgments}

The author is deeply grateful for blood sampling to Dr. Hirotaka Takizawa and Dr. Koichi Kashiwado (Port Square Kashiwado Clinic, Kashiwado Memorial Foundation, Chiba, Japan), Dr. Ikuo Kamitsukasa (Department of Neurology, Chiba Rosai Hospital, Chiba, Japan), Dr. Hideo Shin (Department of Neurosurgery, Higashi Funabashi Hospital, Chiba, Japan), Dr. Takeshi Wada and Dr. Akiyo Aotsuka (Department of Internal Medicine, Chiba Aoba Municipal Hospital, Chiba, Japan), Dr. Eiichiro Nishi and Dr. Mikiko Ohno (Department of Pharmacology, Shiga University of Medical Science, Shiga, Japan), Dr. Minoru Takemoto and Dr. Koutaro Yokote (Department of Clinical Cell Biology and Medicine, Graduate School of Medicine, Chiba University, Chiba, Japan) The authors thank Dr. Sho Takahashi (Clinical Research Center, Chiba University Hospital, Chiba, Japan) for the advice on the statistical analysis and Ms. Keiko Iida (Department of Frontier Surgery,
Graduate School of Medicine, Chiba University, Chiba, Japan) for the support on the experiments. The authors would like to thank Enago (www.enago.jp) for the English language review.

\section{Financial Support}

This work was supported, in part, by research grants from the Japan Agency for Medical Research and Development (AMED) (Practical Research Project for Life-Style related Diseases including Cardiovascular Diseases and Diabetes Mellitus), Japan Science and Technology Agency (JST), and the Ministry of Education, Culture, Sports, Science and Technology (MEXT) in Japan.

\section{Conflicts of Interest Disclosure}

The author declares no conflict of interest.

\section{References}

1) Hao $Y$, Tian $S$, Sun $M$, Zhu $Y$, Nie $Z$, Yang $S$ : Association between matrix metalloproteinase gene polymorphisms and development of ischemic stroke. Int J Clin Exp Pathol 8: 11647-11652, 2015

2) Zhang ZG, Zhang L, Jiang Q, et al.: VEGF enhances angiogenesis and promotes blood-brain barrier leakage in the ischemic brain. J Clin Invest 106: 829-838, 2000

3) Chen B, Zhang F, Li QY, Gong A, Lan Q: Protective effect of Ad-VEGF-bone mesenchymal stem cells on cerebral infarction. Turk Neurosurg 26: 8-15, 2016

4) Goldstein LB, Bushnell CD, Adams RJ, et al.: Guidelines for the primary prevention of stroke: a guideline for healthcare professionals from the American Heart Association/American Stroke Association. Stroke 42: 517-584, 2011

5) Ross R, Glomset JA: The pathogensis of atherosclerosis (first of two parts). N Engl J Med 295: 369-377, 1976

6) Ross R: Atherosclerosis-an inflammatory disease. $N$ Engl J Med 340: 115-126, 1999

7) Jonasson L, Holm J, Skalli O, Bondjers G, Hansson GK: Regional accumulations of T cells, macrophages, and smooth muscle cells in the human atherosclerotic plaque. Arteriosclerosis 6: 131-138, 1986

8) Hansson GK, Holm J, Jonasson L: Detection of activated $\mathrm{T}$ lymphocytes in the human atherosclerotic plaque. Am J Pathol 135: 169-175, 1989

9) Xu QB, Oberhuber G, Gruschwitz M, Wick G: Immunology of atherosclerosis: cellular composition and major histocompatibility complex class II antigen expression in aortic intima, fatty streaks, and atherosclerotic plaques in young and aged human specimens. Clin Immunol Immunopathol 56: $344-359,1990$ 
10) Sahin U, Türeci O, Schmitt H, et al.: Human neoplasms elicit multiple specific immune responses in the autologous host. Proc Natl Acad Sci USA 92: 11810-11813, 1995

11) Chen YT, Scanlan MJ, Sahin U, et al.: A testicular antigen aberrantly expressed in human cancers detected by autologous antibody screening. Proc Natl Acad Sci USA 94: 1914-1918, 1997

12) Chen J, Uchimura K, Stetler RA, et al.: Transient global ischemia triggers expression of the DNA damage inducible gene GADD45 in the rat brain. J Cereb Blood Flow Metab 18: 646-657, 1998

13) Shukla PC, Singh KK, Quan A, et al.: BRCA1 is an essential regulator of heart function and survival following myocardial infarction. Nat Commun 2: 593, 2011

14) Wold MS: Replication protein A: a heterotrimeric, single-stranded DNA-binding protein required for eukaryotic DNA metabolism. Annu Rev Biochem 66: 61-92, 1997

15) Erdile LF, Wold MS, Kelly TJ: The primary structure of the 32-kDa subunit of human replication protein A. J Biol Chem 265: 3177-3182, 1990

16) Martinet W, Knaapen MW, De Meyer GR, Herman AG, Kockx MM: Elevated levels of oxidative DNA damage and DNA repair enzymes in human atherosclerotic plaques. Circulation 106: 927-932, 2002

17) Andreassi MG, Botto N: DNA damage as a new emerging risk factor in atherosclerosis. Trends Cardiovasc Med 13: 270-275, 2003

18) Sweet $T$, Khalili K, Sawaya BE, Amini S: Identification of a novel protein from glial cells based on its ability to interact with NF-kappaB subunits. J Cell Biochem 90: 884-891, 2003

19) Sweet T, Yen W, Khalili K, Amini S: Evidence for involvement of NFBP in processing of ribosomal RNA. J Cell Physiol 214: 381-388, 2008

20) Lacana E, D’Adamio L: Regulation of Fas ligand expression and cell death by apoptosis-linked gene 4 . Nature Med 5: 542-547, 1999

21) Ristić T, Djordjević VB, Deljanin-Ilić M, Cosić V, Kundalić S: Serum Fas/FasL levels in dependence on clinical presentations of coronary disease and their relationship with risk factors. Vojnosanit Pregl 67: 537-542, 2010

22) Niu FN, Zhang X, Hu XM, et al.: Targeted mutation of Fas ligand gene attenuates brain inflammation in experimental stroke. Brain Behav Immun 26: 61-71, 2012

23) Shimokama $T$, Haraoka $S$, Watanabe T: Immunohistochemical and ultrastructural demonstration of the lymphocyte-macrophage interaction in human aortic intima. Mod Pathol 4: 101-107, 1991

24) Ross R: The pathogenesis of atherosclerosis: a perspective for the 1990s. Nature 362: 801-809, 1993

25) Bobryshev YV, Watanabe T: Ultrastructural evidence for association of vascular dendritic cells with
T-lymphocytes and with B-cells in human atherosclerosis. J Submicrosc Cytol Pathol 29: 209-221, 1997

26) Carbone F, Nencioni A, Mach F, Vuilleumier N, Montecucco F: Evidence on the pathogenic role of auto-antibodies in acute cardiovascular diseases. Thromb Haemost 109: 854-868, 2013

27) Goto KI, Sugiyama T, Matsumura R, et al.: Identification of cerebral infarction-specific antibody markers from autoantibodies detected in patients with systemic lupus erythematosus. J Mol Biomark Diagnos 6: 2, 2015

28) Shiratori T, Shimada H, Kagaya A, et al.: Sensitization against anticancer drugs by transfection with UBE21 variant gene into ras-NIH3T3 mouse fibroblasts. Anticancer Res 27: 3227-3233, 2007

29) Kagaya A, Shimada H, Shiratori T, et al.: Identification of a novel SEREX antigen family, ECSA, in esophageal squamous cell carcinoma. Proteome Sci 9: 31, 2011

30) Shimada H, Ito M, Kagaya A, et al.: Elevated serum antibody levels against cyclin L2 in patients with esophageal squamous cell carcinoma. J Cancer Sci Ther 7: 60-66, 2015

31) Linke AT, Marchant B, Marsh P, Frampton G, Murphy J, Rose ML: Screening of a HUVEC cDNA library with transplant-associated coronary artery disease sera identifies RPL7 as a candidate autoantigen associated with this disease. Clin Exp Immunol 126: 173-179, 2001

32) Kaneko M, Ono T, Matsubara T, et al.: Serological identification of endothelial antigens predominantly recognized in Kawasaki disease patients by recombinant expression cloning. Microbiol Immunol 48: 703-711, 2004

33) Kim J, Kim SK, Wang KC, Kim HY, Jeoung D: SEREX identification of the autoantibodies that are prevalent in the cerebrospinal fluid of patients with moyamoya disease. Biotechnol Lett 26: 585-588, 2004

34) Machida T, Kubota M, Kobayashi E, et al.: Identification of stroke-associated-antigens via screening of recombinant proteins from the human expression cDNA library (SEREX). J Transl Med 13: 71, 2015

35) Hiwasa T, Zhang XM, Kimura R, et al.: Association of serum antibody levels against TUBB2C with diabetes and cerebral infarction. Integ Biomed Sci 1: 49-63, 2015

36) Hiwasa T, Machida T, Zhang XM, et al.: Elevated levels of autoantibodies against ATP2B4 and BMP-1 in sera of patients with atherosclerosis-related diseases. Immunome Res 11: 097, 2015

37) Yoshida Y, Wang H, Hiwasa T, et al.: Elevation of autoantibody level against PDCD11 in patients with transient ischemic attack. Oncotarget 9: 8836-8848, 2018

38) Wang H, Zhang XM, Tomiyoshi G, et al.: Association of serum levels of antibodies against MMP1, CBX1, and CBX5 with transient ischemic attack and cerebral infarction. Oncotarget 9: 5600-5613, 2018 
39) Nakamura R, Tomiyoshi G, Shinmen N, et al.: An anti-deoxyhypusine synthase antibody as a marker of atherosclerosis-related cerebral infarction, myocardial infarction, diabetes mellitus, and chronic kidney disease. SM Atheroscler J 1: 1001, 2017

40) Hiwasa T, Tomiyoshi G, Nakamura R, et al.: Serum SH3BP5-specific antibody level is a biomarker of atherosclerosis. Immunome Res 13: 132, 2017

41) Fesmire J, Wolfson-Reichlin M, Reichlin M: Effects of autoimmune antibodies anti-lipoprotein lipase, anti-low density lipoprotein, and anti-oxidized low density lipoprotein on lipid metabolism and atherosclerosis in systemic lupus erythematosus. Rev Bras Reumatol 50: 539-551, 2010

42) Shimada H, Yajima S, Oshima Y, et al.: Impact of serum biomarkers on esophageal squamous cell carcinoma. Esophagus 9: 131-140, 2012

43) Baekkeskov S, Aanstoot HJ, Christgau S, et al.: Identification of the $64 \mathrm{~K}$ autoantigen in insulin-dependent diabetes as the GABA-synthesizing enzyme glutamic acid decarboxylase. Nature 347: 151-156, 1990

44) Johnson SC, Fayed PB, Gorelick PB, et al.: Prevalence and knowledge of transient ischemic attack among US adults. Neurology 60: 1429-1434, 2003

45) Rothwell PM, Warlow CP: Timing of TIAs preceding stroke: time window for prevention is very short. Neurology 64: 817-820, 2005

46) Wang Y, Wang Y, Zhao X, et al.: Clopidogrel with asprin in acute minor stroke or transient ischemic attack. N Engl J Med 369: 11-19, 2013

Address reprint requests to: Yoichi Yoshida, $\mathrm{MD}, \mathrm{PhD}$, Department of Neurological surgery, Graduate School of Medicine, Chiba University, 1-8-1, Inohana, Chuo-ku, Chiba, Chiba 260-8670, Japan. e-mail: y_yoichi0810@chiba-u.jp 